\title{
Experiencia del Centro de Atención a la Mujer, Niños, Niñas y Adolescentes (CAM) ${ }^{1}$
}

\author{
Experience of the Center for Attention to Women, Children, and Adolescents (CAM)
}

Velia Yasideth Sevilla García²

\section{Resumen}

Las mujeres, niños, niñas y adolescentes son los principales afectados de la violencia que caracteriza a nuestros estilos de vida desde hace mucho tiempo. El Centro de Atención a la Mujer, Niños, Niñas y Adolescentes (CAM) es una instancia que surge de la iniciativa de estudiantes de Psicología en Contextos Multiculturales de la Universidad URACCAN, recinto Bluefields, para dar respuesta a las demandas psicológicas específicas que tienen estos grupos sobrevivientes de violencia.

El objetivo de este ensayo es compartir la experiencia de estudiantes de Psicología en Contextos Multiculturales de la URACCAN recinto Bluefields, quienes, inspirados por su compromiso para aportar a un cambio social, han creado el CAM contribuyendo a la solución de una problemática de salud pública en la ciudad de Bluefields.

Palabras clave: Afectaciones psicológicas; CAM; URACCAN; Violencia.

\section{Abstract}

Women, children and teenagers are the main victims of violence, a lifestyle characteristic we have had for a long time. The Attention Center for Women, Children, and Teenagers is an institution that arises from the Psychology in Multicultural Contexts students' initiative of URACCAN University, Bluefields Campus, to give a solution to the psychological problems questions that affect these violence survivor groups.

The objective of this essay is to share the Psychology in Multicultural Contexts students' experience of URACCAN, Bluefields Campus, who, inspired by their commitment to bring about social change, have created the CAM contributing to the solution of a public health problem in Bluefields City.

Keywords: Psychological affectations; CAM; URACCAN; Violence.

\section{Introducción}

Los patrones conductuales que surgen de una sociedad patriarcal y violenta en la cual predomina la expectativa de subordinación de la mujer ante el hombre y la idea de las familias disfuncionales de establecer una educación agresiva como un método de corrección eficaz hacia la niñez y la adolescencia, ha conllevado a que a lo largo de la historia exista una normalización de la violencia dentro de la cotidianidad social. Las relaciones de poder que surgen de las desigualdades son algunos factores que indudablemente

\footnotetext{
$\overline{1}$ XIV Congreso Latinoamericano y del Caribe de Extensión Universitaria y Segundo Congreso Centroamericano de Compromiso Social para la vinculación social de la universidad con la sociedad.

2 Estudiante de Psicología en Contextos Multiculturales, de la Universidad de las Regiones Autónomas de la Costa Caribe Nicaragüense - Recinto Universitario Bluefields. Correo: velsevilla98@gmail.com ORCID: https://orcid.org/0000-0001-7472-4034
}

Recibido: 17/01/2018 - Aprobado: 05/04/2018 
aportan al mantenimiento de este problema y que aún se encuentran presentes en nuestra realidad. La violencia es un problema de salud pública que conlleva a que sus víctimas sufran consecuencias físicas y psicológicas que obstaculizan su desarrollo psicosocial, moral y espiritual óptimo. Pese a los instrumentos legales que protegen los derechos humanos de estos grupos vulnerables, obtenidos de los esfuerzos impulsados por distintos grupos sociales que buscan la igualdad que colabore a mermar esta problemática, la atención psicológica de las víctimas luego de haber culminado un proceso legal no siempre se lleva a cabo. A partir de esta premisa, un grupo de estudiantes de Psicología en Contextos Multiculturales de la Universidad de las Regiones Autónomas de la Costa Caribe Nicaragüense han impulsado la creación de un centro de atención psicológica a mujeres, niños, niñas y adolescentes que cargan con diversos problemas emocionales luego de haber sido víctimas de cualquier tipo de violencia.

Este ensayo titulado: "Experiencia del Centro de Atención a la Mujer, Niños, Niñas y Adolescentes" tiene como objetivo compartir con la comunidad universitaria de URACCAN y demás sujetos de interés, el relato sobre los orígenes e implementación de este proyecto estudiantil que ha sido ideado por estudiantes egresadas y finalistas de la carrera de Psicología en Contextos Multiculturales de URACCAN-Bluefields, con el fin de aportar a la solución al problema de violencia que afectada a nuestra sociedad femenina e infato-juvenil blufileña.

\section{Desarrollo}

Desde tiempos inmemoriales la mujer ha estado en situación de desventaja frente al sexo opuesto, debido a la predominancia cultural de un sistema patriarcal en el que la mujer no se considera como un ser semejante al hombre y por lo cual hemos tenido que estar bajo la tutela y la subordinación de un (padre, hermano, compañero de vida...) siendo un claro reflejo de lo que llamamos "machismo". Así, se ha ido inculcando de generación en generación la imagen de una supremacía masculina en la que subyace la idea de que las mujeres somos propiedades u objetos y por ende, debemos cumplir con los mandatos que establecen los hombres, pues de lo contrario, seremos sancionadas mediante actos de violencia. Todo esto tiene un nombre y es "violencia sexista", la cual a como plantean Blandón, Murguialday \& Vásquez (2011) es una de las más graves manifestaciones de la desigualdad, afecta a las mujeres como colectivo, aunque sea en diferente intensidad y frecuencia.

Paralelamente a esto, vivimos también dentro de una cultura violenta, donde los estilos de crianza seguidos por la mayoría de nuestras familias implementan la violencia como el instrumento clave para la crianza de sus hijos. Así lo confirma también un estudio realizado por Visión Mundial denominado: "Percepción de la Violencia contra la Niñez en Nicaragua", quienes afirman que el hogar y la escuela son los sitios donde los niños, niñas y adolescentes corren más riesgo en este país, pues la población nicaragüense percibe que la escuela y la casa representan un $24 \%$ de riesgo para los niños, niñas y adolescentes (Velásquez, 2018).

Las niñas, niños y adolescentes de Nicaragua tienen problemas y demandas específicas que se derivan de las condiciones de subordinación y discriminación que viven al formar parte de una sociedad sumamente violenta. Aunque la violencia hacia estos sectores puede ser tan común que llega a ser invisible dentro de nuestras sociedades y comunidades, tal a como expuso Zacarías Duarte, Director General del Instituto de Medicina Legal durante la inauguración del foro "Fortalecimiento de la atención interinstitucional de la violencia hacia la niñez y la adolescencia" (Diario metro, 2018), esta no puede ser olvidada ni obviada por quienes la sufren. Los malos tratos físicos y psicológicos hacia la niñez y adolescencia provocan altos niveles de estrés en sus víctimas, y así puede traer graves consecuencias a corto y largo plazo para quienes lo sufren. La Organización Mundial de la Salud (OMS, 2016) plantea que los adultos que han sufrido maltrato en la infancia corren mayor riesgo de sufrir problemas conductuales, físicos y mentales, tales como: depresión, consumo de tabaco, obesidad, comportamientos sexuales de alto riesgo, embarazos no deseados, consumo indebido de alcohol y drogas y actos de violencia (se convierten en víctimas o victimarios). Siempre existe la posibilidad que los niños, niñas y adolescentes que sufren violencia 
reproduzcan estos patrones conductuales abusivos en su presente o en su futuro al interactuar en los diferentes ámbitos de su vida cotidiana, alimentando y perpetuando la cultura violenta. Por tanto, estas situaciones de violencia no sólo afectan a los y las que son "víctimas", sino que nos afecta a todos, pues estas condiciones nos han llevado a aceptar, normalizar y justificar los actos de violencia, lo que implica incluso que debemos quedarnos callados y calladas cuando somos testigos o cuando estamos sometidos a una situación violenta.

Las mujeres, al ser otro grupo altamente vulnerable a sufrir violencia, comparten también las mismas necesidades que la niñez y adolescencia, demandando el fortalecimiento de los factores protectores sociales orientados a erradicar estos niveles de desigualdad que generan violencia.

Tras la lucha continua de las personas que unen esfuerzos para exigir el cumplimiento de los derechos humanos que se les han privado a estos sectores sociales, tales como el movimiento feminista que trabaja para proponer cambios en las relaciones de género mediante el cuestionamiento de la subordinación de las mujeres y el predominio masculino (Blandón et al, 2011, p.11), se ha logrado la creación de instrumentos legales dirigidos a la satisfacción de estas demandas. Dentro de estos instrumentos mencionamos la "Ley integral contra la violencia hacia las mujeres" (Ley 779), a través de la cual se brindan medidas de protección que previenen, sancionan y erradican la violencia en el ámbito público y privado con el objetivo de:

...actuar contra la violencia que se ejerce hacia las mujeres con el propósito de proteger los derechos humanos de las mujeres y garantizarles una vida libre de violencia que favorezca su desarrollo y bienestar conforme a los principios de igualdad y de la no discriminación.

(Asamblea Nacional, 2012)

Por otro lado, garantizando el cumplimiento de los derechos humanos que competen a la niñez y adolescencia, hoy en día contamos con la Ley 870 o "Código de familia", que en el art. 2, inciso "f", establece que los procedimientos planteados en esa ley atienden el interés superior del niño, niña y adolescente, es decir, se prioriza todo aquello que favorezca su pleno desarrollo, físico, psicológico, moral, cultural y social asegurando el disfrute de sus derechos, libertades y garantías de forma integral (Asamblea Nacional de Nicaragua, 2014).

Pese a la existencia de estos instrumentos legales, es aún incuestionable que estos sectores menos favorecidos de nuestras comunidades costeñas y de toda Nicaragua en general aún siguen en la espera de que sus derechos humanos sean cumplidos; lamentablemente, están sometidos a una vida llena de violencia y de negligencias que pueden provocarles incluso sus propias muertes.

En Bluefields han existido organizaciones que se vinculan al trabajo de atención psicosocial de mujeres, niños, niñas, adolescentes y jóvenes que están inmersos en diferentes situaciones de violencia. Entre estas podemos mencionar las siguientes: Centro de Atención Psicosocial (CAPS), encargado de dar atención en salud mental y adiciones al territorio de la Región Autónoma de la Costa Caribe Sur (RACCS) y que dentro de sus servicio se desarrolla un programa de prevención y atención a las víctimas de violencia familiar, disponen de una psicóloga que atiende a mujeres y víctimas de abusos y violencia (Lorente, 2010); y la Comisaría de la Mujer, que era un área administrada por la Policía Nacional para trabajar en pos de atender como es debido las denuncias que las mujeres presentan sobre casos de violencia contra ellas o en detrimento de los niños (La voz del sandinismo, 2015), pero lamentablemente esta atención ya no se da en una estructura especial para la mujer y la niñez, pues las denuncias por violencia se reciben ahora en las instalaciones de Auxilio Judicial de la Policía Nacional. Es importante mencionar que aunque existen organismos como estos, no toda la población conoce con claridad los mecanismos u organizaciones que deben visitar para ser asistidas cuando sufren de violencia.

La ley 779 en su arto. 19 nos dice que el Estado tiene la obligación de proteger a las víctimas (ahora denominadas sobrevivientes), así como de garantizar su seguridad. Dentro de las medidas que se brindan 
para garantizar dichos factores, la ley establece que se les debe de proporcionar servicios de atención, asesoría jurídica y tratamiento psicológico, especializado y gratuito (Asamblea Nacional, 2012). En línea con el cumplimiento de lo establecido en la ley, el Ministerio Público a través de la Unidad Especializada de Atención a Víctimas les brinda un acompañamiento jurídico, así como un acompañamiento psicológico a las víctimas que se sienten inestable emocionalmente, al momento de ir a declarar al juicio oral y público (Ministerio Público de Nicaragua, 2016). Sin embargo, no siempre se les brinda un seguimiento psicológico luego de haber concluido el proceso legal que les ayude a sanar las secuelas emocionales que les han dejado sus experiencias traumáticas.

Por otro lado, existen casos de sobrevivientes de violencia que aún no se sienten preparadas para llevar a cabo un proceso jurídico en contra de su agresor, pero desean tener ayuda psicológica. Tras esta situación, un grupo de estudiantes de Psicología en Contextos Multiculturales de la Universidad de las Regiones Autónomas de la Costa Caribe Nicaragüense (URACCAN), decidimos crear un centro de atención psicológica para atender a estos grupos vulnerables a la violencia, mediante la implementación de diferentes metodologías dirigidas a la concientización, sensibilización, tratamiento y prevención de la violencia en nuestra ciudad de Bluefields, encaminándonos a aportar a esta situación que es, tal a como lo plantea Baca (2013), un problema de salud pública.

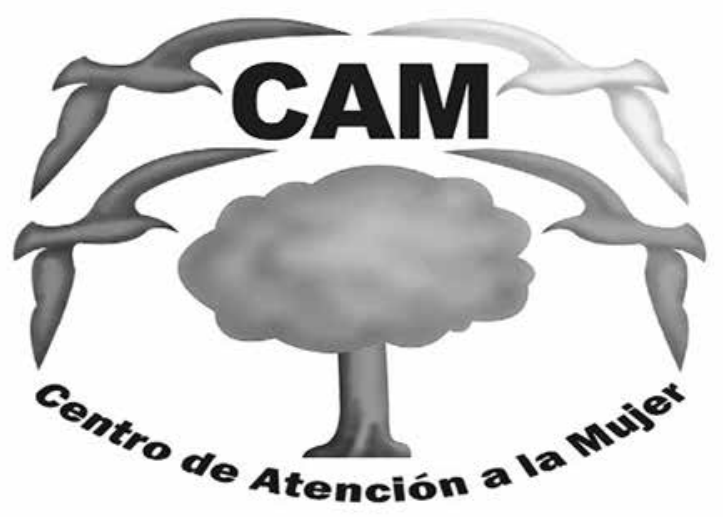

Figura 1. Logotipo del CAM.

El Centro de Atención a la Mujer, Niños, Niñas y Adolescentes (CAM) nació como iniciativa de estudiantes de la carrera de Psicología, luego de haber participado en una conferencia dirigida por el psicólogo español Salvador Badía, en la que se abordaban los problemas de violencia en la ciudad de Bluefields y en el Caribe Sur, haciendo énfasis en el abuso sexual y las secuelas que este deja en sus sobrevivientes. En esta actividad se evidenció por testimonios de los y las estudiantes partícipes que la violencia era un problema que se vive en la ciudad y en múltiples escenarios (en la escuela, en la casa, en la calle, en el trabajo, etc.) siendo las principales víctimas las mujeres, niños, niñas y adolescentes, quienes sufren efectos psicológicos negativos colaterales tras estos hechos.

Partiendo de la observación de estas situaciones de violencia a nivel del municipio de Bluefields y otros municipios de la región, donde las personas sobrevivientes son en su mayoría mujeres, niños, niñas y adolescentes, las estudiantes de la carrera de Psicología en Contextos Multiculturales de URACCAN: Velia Sevilla, Yonaira Martínez, Kimberly Castillo, Jessenia Cáceres y Virginia Murillo consideramos la necesidad de crear un centro de atención psicológico destinado exclusivamente para la atención de mujeres, niños, niñas y adolescentes sobrevivientes de violencia. Así, decidimos poner en práctica nuestros conocimientos científicos y siendo acompañadas por el Msc. Luis Barrera y la Msc. Ilenia García, docentes de la carrera, nos organizamos junto con Salvador Badía y las autoridades de la Catedral Nuestra Señora del Rosario de Bluefields, para contribuir en la atención de esta problemática que afecta no sólo a sus víctimas, sino también a toda la sociedad en general. 


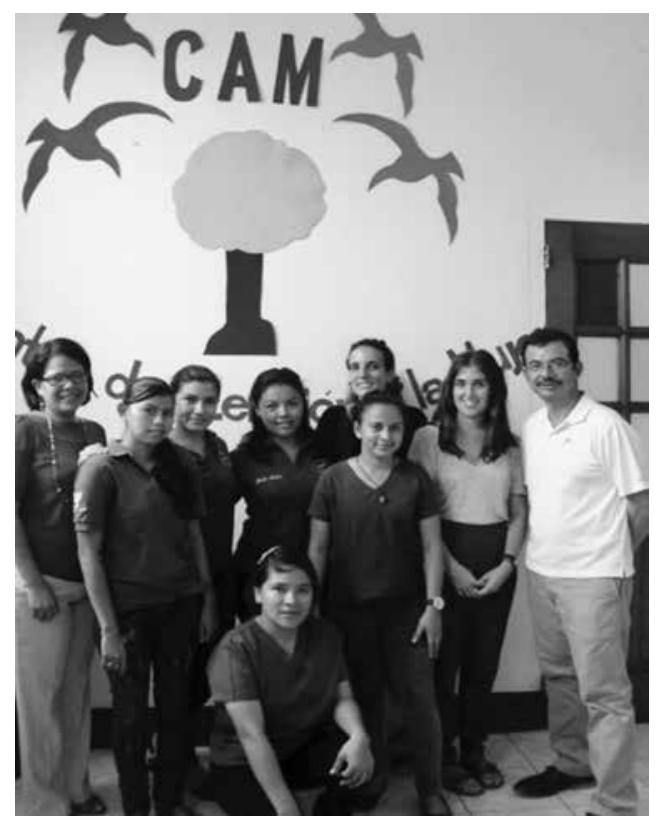

Figura 2. Estudiantes y docentes fundadores de CAM, acompañados de psiquiatras españolas

Posterior a una coordinación entre la universidad URACCAN, la Iglesia Católica, especialistas voluntarios españoles de la Salud Mental y docentes de la carrera de Psicología, el CAM fue inaugurado el día o5 de agosto del año 2016 con el objetivo de brindar acompañamiento psicológico a la mujer, niñez y adolescencia en la atención de problemas psicosociales asociados a la violencia en la ciudad de Bluefields. Desde su inauguración hasta mediados del año 2017, se brindó atención de forma continua todos los miércoles de las 8:00 a.m. a las 12:00 m. y de la 2:00 pm a las 5:00 p.m. en las oficinas de la Casa Cural de la Catedral Nuestra Señora del Rosario de la ciudad de Bluefields. Luego, las instalaciones del CAM fueron trasladadas a URACCAN recinto Bluefields. Desde su fundación hasta la actualidad, el funcionamiento del centro ha estado a cargo de las impulsoras del proyecto, de las cuales tres han egresado de la carrera y dos se encuentran cursando el cuarto año de la carrera.

El CAM ha incluido en sus programas de intervención diversas actividades dirigidas no solamente a la atención psicológica individual, sino también a la concientización, sensibilización, y prevención de la violencia, educando a la población de Bluefields sobre diversas temáticas psicosociales que de una u otra forma se vinculan a los problemas de violencia.

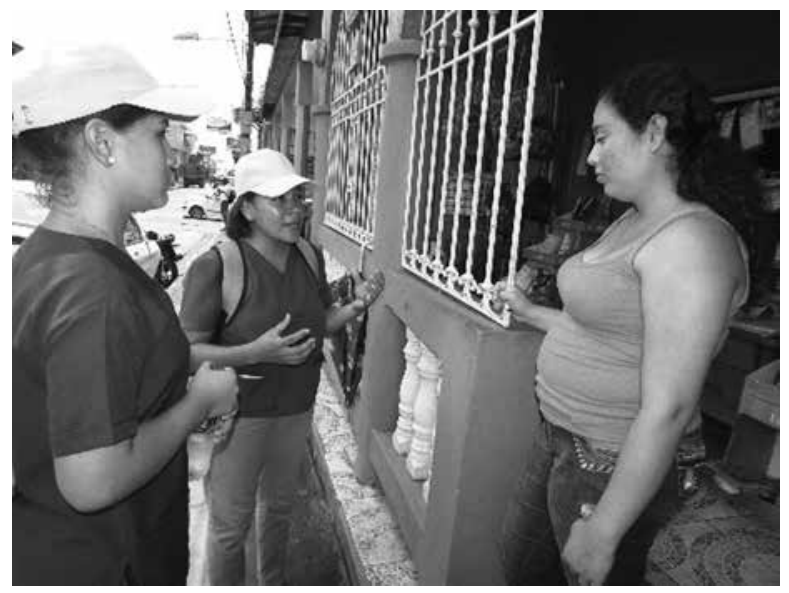

Figura 3. Estudiantes de CAM haciendo visitas domiciliares de concientización. Fuente: Noticias de Bluefields. 
Para cumplir con nuestros objetivos, el CAM realiza las siguientes actividades:

1. Atención psicológica individual, llevada a cabo por docentes y estudiantes egresadas de Psicología.

2. Visita casa a casa en barrios del sector urbano de Bluefields para facilitar charlas educativas donde se aborda el tema de la violencia de género, violencia intrafamiliar, la importancia de la prevención de todo tipo de violencia y de promover la comunicación asertiva intrafamiliar.

3. Programa radial "Mujeres valiosas", en el que se abordan temas de interés psicosocial, brindando medidas de prevención referentes a diversas temáticas. Este ha sido dirigido por las estudiantes del tercer año de Psicología, con el acompañamiento del docente Luis Barrera y transmitido de 6:00 am a 7:00 am en la radio URACCAN Bluefields.

4. Visitas a las escuelas de la ciudad de Bluefields, con el objetivo de compartir con los estudiantes información relacionada a la violencia y para captar a aquellos que requieran y deseen recibir atención psicológica en el centro.

Además, dentro de sus servicios, el CAM provee información, orientación y asesoramiento a mujeres, niños/as y adolescentes con problemas de violencia, así como a sus familias, psicoterapia individual y familiar, así como la facilitación de procesos de capacitación en temas relacionados a la violencia de género e intrafamiliar. Es importante mencionar que este centro ha sido también un centro de referencia para demás estudiantes de psicología que detectan casos de violencia en los que los sobrevivientes requieren o solicitan atención especializada durante las prácticas comunitarias, remitiéndolos a nuestras instancias.

Durante su existencia, el CAM obtuvo los siguientes resultados:

\begin{tabular}{|l|c|}
\hline \multicolumn{1}{|c|}{ Actividades } & Cantidades \\
\hline Atención Directa & 73 \\
\hline Casos atendidos & 18 \\
\hline Atenciones psicológicas domiciliares & 6 \\
\hline Barrios visitados & 7 \\
\hline Casas visitadas & 212 \\
\hline Programas Radiales & 17 \\
\hline Escuelas visitadas & 2 \\
\hline
\end{tabular}

Luego año y medio de funcionamiento, el CAM como proyecto estudiantil dejó de prestar sus servicios temporalmente, ya que está en un proceso de restructuración para convertirse en un centro de atención integral a las mujeres, niñez y adolescencia sobrevivientes de violencia autorizado por el SILAIS. Actualmente, a más de dos años de la fundación de CAM, las estudiantes mantenemos vivo el compromiso social de seguir aportando a esta situación, siendo persistentes en nuestra labor y en la búsqueda de optimizar nuestros servicios y poder alcanzar nuestra visión de ser un centro comunitario e intercultural de referencia especializado en la atención psicológica integral para mujeres, niños, niñas y adolescentes víctimas de violencia de la ciudad de Bluefields. Actualmente, este proyecto que nació como una iniciativa de cinco estudiantes que deseábamos aportar al cambio de esta situación está en proceso de establecerse como el primer laboratorio académico para el fortalecimiento teórico - práctico de estudiantes de la carrera de psicología y sociología de la URACCAN. 


\section{Conclusiones}

Desde sus inicios y como resultado del arduo trabajo realizado por el equipo de docentes y estudiantes, el CAM logró evolucionar de ser conocido como un "simple proyecto donde trabajan los estudiantes" a establecerse como un centro de referencia para los pobladores de la ciudad de Bluefields que brinda atención psicológica a las mujeres, niños, niñas y adolescentes sobrevivientes de violencia. De esta manera, se atendieron diversos casos de violencia intrafamiliar, de género y social. A través de nuestro programa radial transmitido en la frecuencia de Radio URACCAN Bluefields (91.1 FM) logramos alcanzar los hogares de nuestra población blufileña informando a los ciudadanos sobre diversos temas de interés psicosocial, dentro de los cuales podemos mencionar el suicidio, la violencia intrafamiliar, el bullying y el manejo correcto de redes sociales. Es conveniente mencionar la importancia de la presencia de docentes y autoridades de la Universidad de las Regiones de la Costa Caribe Nicaragüense, quienes nos han abierto los espacios para compartir nuestro proyecto y quienes han aportado al mantenimiento del CAM.

Actualmente, el CAM se encuentra en un proceso de transformación para continuar con su funcionamiento como un centro de referencia para la atención a los casos de violencia. Como estudiantes, mantenemos la motivación para seguir trabajando para alcanzar nuestros proyectos a futuro encaminados al fortalecimiento del CAM como un centro líder en atención psicológica para las mujeres, niños, niñas y adolescentes que se ven afectados por la violencia y como un centro de formación a estudiantes de ciencias humanísticas que deseen perfilarse profesionalmente para contribuir a la solución de esta problemática.

\section{Lista de referencia}

Asamblea Nacional (2012). Ley No. 779. Ley Integral Contra la Violencia hacia las Mujeres y de Reforma a la Ley No. 641, "Código Penal". Publicada en La Gaceta Diario Oficial No. 35, del 22 de febrero del 2012. Nicaragua.

Asamblea Nacional. (2014). Ley No. 870. Código de Familia. Publicada en La Gaceta No. 190 del 8 de Octubre de 2014. Nicaragua.

Baca Suárez, M. (2013). OMS: violencia de género ya alcanza niveles de "pandemia". Poder Judicial: Dirección General de Comunicación. Recuperado de: https://www.poderjudicial.gob.ni/prensa/notas_prensa_detalle.asp?id_noticia $=3645$

Blandón Gadea, M., Murguialday Martínez, C. \& Vásquez García, N. (2011). Los cuerpos del Feminismo Nicaragüense. Recuperado de: http://biblioteca.hegoa.ehu.es/downloads/19001/\%2Fsystem\%2Fpdf\%2F2906\%2Flos_cuerpos_del_feminismo_Nicaraguense.pdf

Ministerio Público de Nicaragua (2016). Unidad Especializada de Atención a Víctimas. Recuperado de: https://ministeriopublico.gob.ni/oficinas-de-atencion-a-las-victimas/

Organización Mundial de la Salud (2016). Maltrato infantil. Recuperado de: http://www.who.int/es/ news-room/fact-sheets/detail/child-maltreatment

S.A. (2018, 6 de abril). Combatir la violencia en niñez y adolescencia Unicef. Diario metro. Recuperado de: http://diariometro.com.ni/nacionales/171415-unicef-violencia-ninos-adolecentes-ninez-nicaraguenses/

S.A. (2015, 27 de julio). Comisaría de la Mujer, sinónimo de seguridad. La voz del sandinismo. Recuperado de: http://www.lavozdelsandinismo.com/nicaragua/2015-07-27/ comisaria-de-la-mujer-sinonimo-de-seguridad/

Velásquez, U. (2018, 31 de enero). Estudio revela los sitios en Nicaragua donde la niñez está más expuesta a la violencia. El Nuevo Diario. Recuperado de: https://www.elnuevodiario.com.ni/ nacionales/454419-estudio-revela-sitios-nicaragua-donde-ninez-esta-m/ 